\title{
Tratamento da necrose aguda de retina: revisão sistemática
}

\author{
Treatment of acute retinal necrosis: systematic review
}

\author{
Moysés Eduardo Zajdenweber ${ }^{1}$ \\ Cristina Muccioli ${ }^{2}$
}

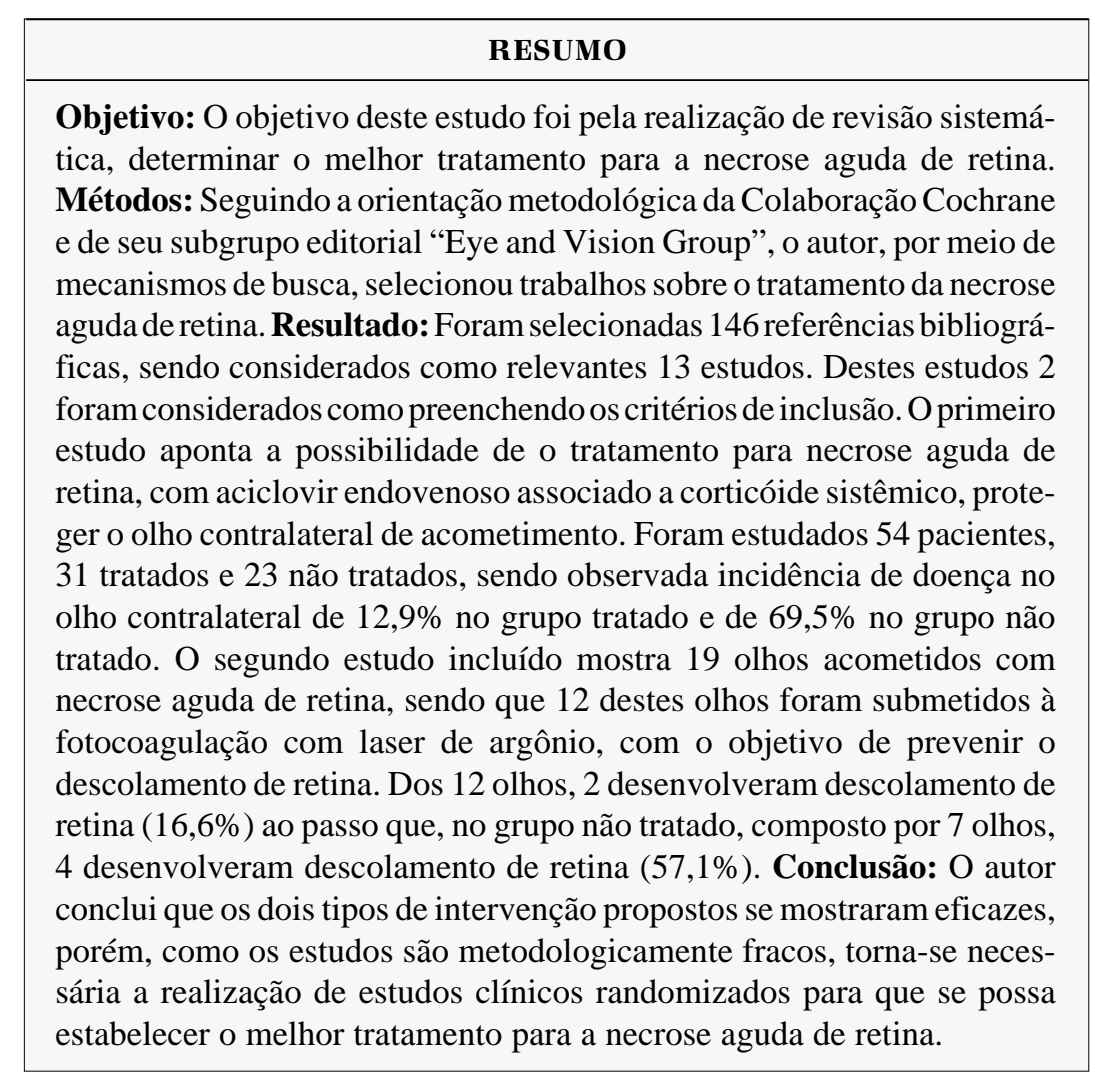

Descritores: Síndrome de necrose retiniana aguda/quimioterapia; Retina/patologia; Aciclovir/ uso terapêutico; Medicina baseada em evidencia; Resultado de tratamento

\section{INTRODUCÃO}

Podemos definir a necrose aguda de retina como uma vasculite oclusiva severa que primariamente afeta as artérias retinianas e coroidianas, levando ao desenvolvimento de retinite necrozante ${ }^{(1)}$.

A necrose aguda de retina foi primeiramente descrita por Urayama no Japão, há cerca de 30 anos, e foi inicialmente por ele chamada de uveíte de Kirisawa, como uma homenagem a seu professor Nagonori Kirisawa. Nesta ocasião foram relatados seis casos de uveíte unilateral difusa, periarterite e áreas de necrose da retina periférica acompanhada de descolamento de retina $^{(2)}$.

O primeiro relato de necrose aguda de retina na literatura americana foi em $1977^{(3)}$. Em 1978 Young e Bird utilizaram pela primeira vez o termo necrose aguda de retina bilateral ${ }^{(4)}$ e apenas em 1980 Hayreh et al. consoli- 
daram o termo necrose aguda de retina em trabalho apresentado no congresso da $\mathrm{ARVO}^{(5)}$.

No Brasil os primeiros casos da doença foram publicados por Sato et al. em 1985, sendo que os autores descreveram dois casos da doença ${ }^{(6)}$. Amaro e Amaro em 1987, relatam 1 caso de necrose aguda de retina, presumindo possível etiologia viral para a doença ${ }^{(7)}$. Em 1991, em outra série, Medeiros e Medeiros apresentaram 2 casos, sendo um deles em paciente com AIDS $^{(8)}$ e em 1993 Lacerda descreveu 12 casos da doença, todos em indivíduos imunocompetentes ${ }^{(9)}$.

A necrose aguda de retina tem distribuição mundial e pode ocorrer em qualquer idade ou sexo, havendo a possibilidade de acometer os dois olhos; porém, na maioria dos casos, é inicialmente unilateral. Embora esta patologia, a princípio tenha sido descrita em indivíduos imunocompetentes, atualmente é, descrita com acometendo indivíduos com imunossupressão de qualquer natureza, como, por exemplo pacientes com síndrome de imunodeficiência adquirida (AIDS) ${ }^{(10)}$.

Observação de agentes patológicos, anticorpos intra-oculares e testes como o PCR (Polimerase chain reaction), identificaram os vírus varicela zoster, herpes simples tipo 1 e 2 e raramente o citomegalovírus como potenciais causadores da necrose aguda de retina ${ }^{(1)}$.

Embora seja possível identificar a causa da doença, o diagnóstico da necrose aguda de retina deve ser baseado em critérios clínicos. Em 1994 Holland e a American Uveitis Society estabeleceram estes critérios $^{(11)}$ : A designação necrose aguda de retina deve se basear somente em seu aspecto clínico e no curso da infecção. As características clínicas que devem ser observadas incluem: áreas focais, bem demarcadas de necrose da retina localizadas na periferia da mesma (primariamente envolvendo área adjacente, ou, externamente, a arcada vascular temporal); progressão rápida da necrose (se terapia antiviral não for estabelecida); evidência de vasculopatia oclusiva com envolvimento arteriolar; reação inflamatória significativa em câmara anterior e vítreo; características que confirmam, mas não são obrigatórias, incluem: neuropatia ou atrofia óptica; esclerite e dor; sendo o diagnóstico baseado em aspectos clínicos e no curso da doença, o termo necrose aguda de retina não é influenciado pelo isolamento de qualquer vírus ou outro patógeno de qualquer fluido ou tecido ocular.

A necrose aguda de retina geralmente se inicia com desenvolvimento de lesões branco-amareladas, redondas ou ovais, de retinite, localizadas no epitélio pigmentário ou retina profunda em sua porção pós-equatorial. A necrose da retina pode ficar restrita a um quadrante, ou menos, ou comprometer vários quadrantes, confluindo na periferia e progredindo até atingir o pólo posterior. A vitreíte costuma aumentar com a evolução da doença, podendo chegar a uma intensidade que dificulta a observação das estruturas do fundo de olho. Com a progressão da necrose retiniana e da inflamação vítrea, pode surgir, semanas ou meses após o início do quadro, descolamento regmatogênico de retina, que geralmente acomete mácula e grandes áreas da retina, sendo a principal causa de baixa de acuidade visual decorrente desta doença, assim como a sua complicação mais comum, podendo ocorrer em 24 a $80 \%$ dos $\operatorname{casos}^{(6-12)}$.

A inflamação ocular associada à necrose aguda de retina geralmente é autolimitada e em casos não tratados o desaparecimento da inflamação intra-ocular ocorre entre 6 e 12 semanas após o início dos sintomas ${ }^{(13)}$.

O diagnóstico diferencial da necrose aguda de retina inclui: endoftalmite, síndrome de Behçet, uveíte intermediária, toxoplasmose, sífilis, retinite por citomegalovírus, sarcoidose, linfoma intra-ocular e necrose progressiva da retina externa $(\mathrm{PORN})^{(10)}$.

As opções de tratamento para necrose aguda de retina incluem terapia antiviral com aciclovir, valaciclovir ou famciclovir ${ }^{(14)}$. Outra possibilidade é o uso de corticosteróides, fotocoagulação com laser de argônio e tratamento cirúrgico com vitrectomia via pars plana. Atualmente, o tratamento de escolha é aciclovir endovenoso $10 \mathrm{mg} / \mathrm{kg}$ de $8 / 8$ horas de 10 a 14 dias, seguido de aciclovir oral $800 \mathrm{mg}$ a cada 5 horas por no mínimo 3 meses ${ }^{(15)}$. Corticóides por via oral são freqüentemente usados para diminuir a inflamação. Se a doença é severa foscarnet ou ganciclovir podem ser utilizados ${ }^{(16)}$. Na presença de descolamento de retina, cirurgia de vitrectomia via pars plana com fotocoagulação a laser de argônio e óleo de silicone deve ser realizada ${ }^{(17)}$.

O objetivo deste trabalho é, através de revisão sistemática, determinar o melhor tratamento para a necrose aguda de retina.

\section{MÉTODOS}

\section{Características dos estudos para revisão}

\section{Tipos de estudos}

O padrão ouro para uma revisão sistemática que aborda aspectos de terapêutica deve ter como critério de inclusão estudos clínicos randomizados cegos ou duplo cegos.

$\mathrm{Na}$ ausência destes estudos, o autor considerou, como aptos para preencher os critérios de inclusão, estudos clínicos controlados, independente da forma de distribuição dos grupos.

\section{Participantes}

Pessoas de qualquer sexo ou idade, portadores de necrose aguda de retina, com ou sem síndrome de imunodeficiência adquirida (AIDS), diagnosticada segundo critérios clínicos estabelecidos em 1994 por Holland e a American Uveitis Society ${ }^{(11)}$.

\section{Tipos de intervenção}

Qualquer intervenção, farmacológica ou cirúrgica, para o tratamento da necrose aguda de retina foi avaliada.

\section{Tipos de desfechos}

Os desfechos estudados foram divididos em dois grupos:

- Primários: melhora da atividade inflamatória; melhora da acuidade visual. 
- Secundários: complicações (descolamento de retina, catarata, glaucoma, atrofia óptica); efeitos colaterais; relacionados à qualidade de vida.

\section{Estratégia de busca para identificação dos estudos}

Realizada pesquisa em bases de dados virtuais: CENTRAL (the Cochrane controlled trials register), MEDLINE (Medical Literature, Analysis and Retrieval System on line), EMBASE (Excerpta Médica Database) e LILACS (Literatura Latino Americana de Ciências da Saúde).

Busca manual também foi realizada. Foram checadas pelo autor referências bibliográficas em artigos identificados. Foi ainda investigada junto à indústria farmacêutica, a existência de estudos em andamento ou não publicados. Não houve restrição quanto à língua nesta pesquisa.

\section{Métodos da revisão}

O autor avaliou os resumos obtidos através das diferentes estratégias de busca e os classificou em:

a) elegíveis: estudos que apresentam relevância e têm possibilidade de serem incluídos na revisão.

b) não elegíveis: estudos sem relevância, sem possibilidade de inclusão na revisão sistemática.

\section{Avaliação da qualidade metodológica}

A qualidade metodológica dos estudos foi avaliada pelo autor, seguindo orientação do manual para revisão da Colaboração Cochrane (Cochrane Reviewer's Handbook) ${ }^{(18)}$. Quatro parâmetros foram considerados: ocultação da alocação, método de alocação, documentação de exclusão e seguimento clínico completo.

Cada parâmetro foi graduado em: A) adequado; B) pouco claro C) inadequado; D) não randomizado.

\section{Coleta dos dados}

$\mathrm{O}$ autor coletou os dados utilizando, metodologia descrita pelo "Cochrane Eyes and Vision Group".

O grupo orienta a extração dos dados relevantes dos desfechos estabelecidos, no sentido de que ela seja feita em separado para cada estudo. Para dados dicotômicos, dados em que apenas dois desfechos são possíveis, deve-se estabelecer o número de pacientes determinado para o evento, e o número de pacientes submetidos ao evento. Para dados contínuos se consideram a média e o desvio padrão.

\section{Análise dos desfechos}

Quanto à inflamação intra-ocular, foi considerada a presença de atividade inflamatória de câmara anterior ou de cavidade vítrea ao início do tratamento e a melhora da condição ao término deste. Os resultados relativos à acuidade visual foram avaliados, considerando o início e o fim do tratamento, tendo sido usada tabela de Snellen convertida. Complicações como descolamento de retina, catarata, glaucoma e atrofia óptica foram analisadas, considerando a presença ou ausência destas condições. Efeitos colaterais, caso presentes, seriam analisados como Risco Relativo para um ou mais eventos isoladamente.

\section{Síntese dos dados}

Os dados extraídos dos estudos foram todos dicotômicos, portanto os resultados foram expressos em Risco Relativo (proporção de eventos no grupo tratamento em relação à proporção de eventos no grupo controle), sendo que seu respectivo intervalo de confiança foi de $95 \%$.

\section{RESULTADOS}

Foram obtidas, através dos diferentes sistemas de busca, 146 referências, sendo que 13 estudos foram considerados elegíveis, relevantes, entre os quais 2 preencheram os critérios de inclusão para a revisão. Os demais foram excluídos por se tratarem de relato de caso, ou série de casos sem grupo controle.

Em um estudo clínico controlado, Palay et al. (1991) relacionam o uso de aciclovir endovenoso (EV) como fator de proteção contra o aparecimento da doença no olho contralateral ${ }^{(19)}$.

Estudaram 54 pacientes, que apresentaram necrose aguda de retina unilateral, sendo $32(59,2 \%)$ do sexo masculino e 22 $(40,8 \%)$ do sexo feminino. Divididos em dois grupos, o primeiro grupo ficou composto por 31 pacientes $(57,4 \%)$, sendo 21 do sexo masculino $(67,7 \%)$ e 10 do sexo feminino (32,3\%), com média de idade de 43 anos. Neste grupo os pacientes foram tratados com aciclovir EV (1.500 mg/kg/dia), por 7 a $10 \mathrm{dias,}$ seguido de aciclovir oral, $800 \mathrm{mg}, 5$ vezes por dia, por 2 a 4 semanas; $23(74,2 \%)$ pacientes deste grupo usaram corticosteróide sistêmico associado. O segundo grupo foi formado por 23 pacientes $(42,6 \%), 11$ do sexo masculino $(52,2 \%)$ e 12 do sexo feminino $(47,8 \%)$, com média de idade de 41 anos. Os pacientes deste grupo não receberam tratamento com aciclovir e $14(60,8 \%)$ usaram corticosteróide sistêmico.

Dos 31 pacientes tratados com aciclovir $27(87,1 \%)$ não desenvolveram a doença no olho contralateral, sendo que 15 desses 27 pacientes $(55,6 \%)$ foram examinados por no mínimo um ano, após início do quadro e $6(22,2 \%)$, por no mínimo dois anos após o início do quadro.

Dos 23 pacientes que não fizeram uso de aciclovir, somente $7(30,4 \%)$ não desenvolveram a doença no olho contralateral e 3 deles $(42,8 \%$ ) foram examinados por no mínimo um ano, após início do quadro e $3(42,8 \%)$, por no mínimo dois anos após o início do quadro.

A análise estatística destes dados mostrou um risco relativo de 0,19 com intervalo de confiança de $95 \%(0,07$ a 0,48$)$, favorecendo o tratamento (Gráfico 1).

O tempo de acompanhamento médio do grupo tratado foi de 12 meses e o tempo médio do grupo que não usou aciclovir foi de 11 meses.

O segundo estudo incluído na revisão é um estudo clínico controlado, em que Sternberg et al. (1988) propõem a fotocoa- 
gulação com laser de argônio como fator de proteção contra o descolamento de retina em pacientes com diagnóstico clínico de necrose aguda de retina ${ }^{(20)}$. São comparados 2 grupos, no total de 16 pacientes e 19 olhos, sendo o primeiro grupo de 12 olhos $(63,1 \%)$ submetido à fotocoagulação com laser de argônio: 6 destes olhos submetidos ao procedimento logo que realizado o diagnóstico e 6 após inicio de tratamento com aciclovir EV e corticóide via oral. O segundo grupo, composto por 7 olhos $(36,9 \%)$, não foi submetido à fotocoagulação com laser de argônio. Todos os pacientes, dos dois grupos, foram submetidos ao tratamento clínico com aciclovir inicialmente EV e em seguida por via oral.

No grupo tratado, 2 (17\%) dos 12 olhos desenvolveram descolamento de retina, sendo os pacientes acompanhados por 18 meses em média (4-36 meses). No grupo não tratado, 4 $(57,1 \%)$ dos 7 olhos desenvolveram descolamento de retina.

A análise estatística destes dados mostrou um risco relativo de 0,35 com intervalo de confiança de $95 \%(0,09$ a 1,41), favorecendo o tratamento (Gráfico 2).

Os resultados estão resumidos nas tabelas 1 e 2 .

\begin{tabular}{|c|c|c|c|c|}
\hline \multirow{3}{*}{$\begin{array}{l}\begin{array}{l}\text { Comparação: Ac } \\
\text { Desfecho: }\end{array} \\
\text { Ost } \\
\text { Estudo } \\
\text { Palay et al., 1991 } \\
\end{array}$} & \multicolumn{2}{|c|}{$\begin{array}{l}\text { EV } X \text { sem tratamento } \\
\text { tralateral sem doença }\end{array}$} & & \multirow{3}{*}{$\begin{array}{c}\begin{array}{c}\text { RR } \\
\text { (95\%IC Fixado) }\end{array} \\
0,19(0,07-0,48)\end{array}$} \\
\hline & $\begin{array}{c}\text { Tratamento } \\
\mathrm{n} / \mathrm{N}\end{array}$ & $\begin{array}{c}\text { Controle } \\
n / N\end{array}$ & $(95 \%$ & \\
\hline & $4 / 31$ & $16 / 23$ & $\leftrightarrow$ & \\
\hline
\end{tabular}

RR= risco relativo; $95 \%$ IC Fixado= intervalo de confiança de $95 \%$; $=$ amostra que não atingiu desfecho desejado em cada grupo; $N=$ total da amostra em cada grupo de intervenção.

Gráfico 1 - Comparação de pacientes usando aciclovir em pacientes sem tratamento

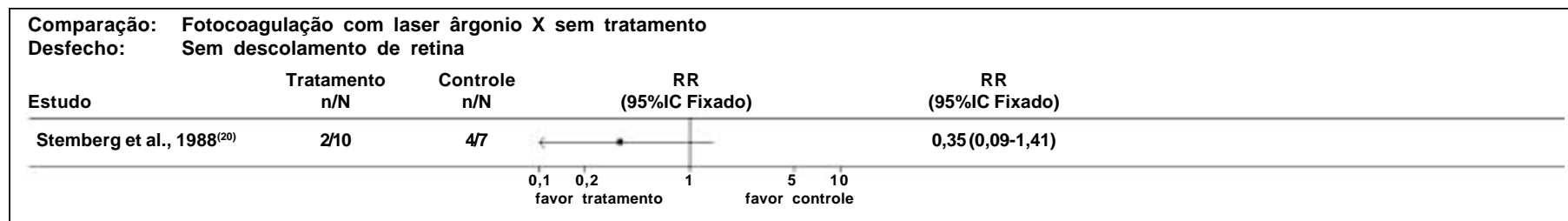

RR= risco relativo; $95 \%$ IC Fixado= intervalo de confiança de $95 \% ; n=$ amostra que não atingiu desfecho desejado em cada grupo; $N=$ total da amostra em cada grupo de intervenção.

Gráfico 2 - Comparação de pacientes submetidos à fotocoagulação com pacientes sem tratamento

\begin{tabular}{|c|c|c|c|c|}
\hline & & Tabela 1. Tabela de estudos i & Iuídos & \\
\hline Estudo & Método & Participantes & Intervenções & Desfechos \\
\hline $\begin{array}{l}\text { Palay, et al. } \\
1991\end{array}$ & $\begin{array}{l}\text { Estudo clínico controlado } \\
\text { não randomizado } \\
\text { Estudo multicêntrico } \\
\text { com pacientes incluídos } \\
\text { nos seguintes centros: } \\
\text { Bascom Palmer Eye } \\
\text { Institute, the Emory } \\
\text { Eye Center, the Jules } \\
\text { Stein Eye Institute, the } \\
\text { Medical College of } \\
\text { Wisconsin, the Wilmer } \\
\text { Eye Institute }\end{array}$ & $\begin{array}{l}\mathrm{n}=54 \text { pacientes com necrose } \\
\text { aguda de retina unilateral sem } \\
\text { AIDS ou imunossupressão } \\
\text { por outras causas } \\
31 \text { pacientes tratados com } \\
\text { aciclovir ( } 21 \text { masculino, } \\
10 \text { feminino; idade média } \\
43 \text { anos) } \\
23 \text { pacientes não tratados } \\
\text { com aciclovir ( } 11 \text { masculino, } \\
12 \text { feminino; idade média } \\
41 \text { anos) }\end{array}$ & $\begin{array}{l}\text { Aciclovir intravenoso } \\
(1.500 \mathrm{mg} / \mathrm{kg} / \mathrm{dia}) 7-10 \text { dias } \\
\text { aciclovir oral } 800 \mathrm{mg} / 5 \mathrm{x} \text { dia } \\
2-4 \text { semanas } \\
\text { Corticosteróide sistêmico } \\
(\mathrm{n}=31) \\
\text { Sem tratamento } \\
(\mathrm{n}=23)\end{array}$ & $\begin{array}{l}\text { Olho contralateral sem } \\
\text { sinais da doença com } \\
\text { acompanhamento } \\
\text { médio de } 12 \text { meses }\end{array}$ \\
\hline $\begin{array}{l}\text { Sternberg, et al. } \\
1988\end{array}$ & $\begin{array}{l}\text { Estudo clínico controlado } \\
\text { não randomizado } \\
\text { Estudo multicêntrico } \\
\text { Com pacientes incluídos } \\
\text { nos seguintes centros: } \\
\text { the Emory EyeCenter, } \\
\text { the Medical College of } \\
\text { Wisconsin, the Wilmer } \\
\text { Eye Institute, Univesity } \\
\text { of Louisville }\end{array}$ & $\begin{array}{l}\mathrm{n}=19 \text { olhos com necrose } \\
\text { aguda de retina } \\
12 \text { olhos submetidos à } \\
\text { fotocoagulação com laser } \\
\text { de argônio } \\
7 \text { olhos não submetidos à } \\
\text { fotocoagulação com laser } \\
\text { de argônio }\end{array}$ & $\begin{array}{l}\text { Fotocoagulação com laser } \\
\text { de argônio }\end{array}$ & $\begin{array}{l}\text { Ausência de } \\
\text { descolamento de } \\
\text { retina }\end{array}$ \\
\hline
\end{tabular}




\begin{tabular}{|c|c|c|}
\hline \multicolumn{3}{|c|}{ Tabela 2. Tabela de estudos excluídos } \\
\hline Estudo & Intervenções & Razões para exclusão \\
\hline Aslanides et al., 2002 & Valaciclovir + corticóide oral + fotocoagulação & Série de casos, sem grupo controle \\
\hline Müller, Velhagen, Pleyer, 2000 & $\begin{array}{l}\text { Aciclovir EV + vitrectomia via pars plana (VVPP) } \\
+ \text { implante de óleo de silicone }\end{array}$ & Série de casos, sem grupo controle \\
\hline Luu et al., 2000 & $\begin{array}{l}\text { Aciclovir oral, EV e intravítreo + corticóide oral e EV + } \\
\text { foscarnet EV + ganciclovir EV + fotocoagulação com laser } \\
\text { de argônio }\end{array}$ & Série de casos, sem grupo controle \\
\hline $\begin{array}{l}\text { Hudde, Althaus, } \\
\text { Sundmacher, } 1998\end{array}$ & Aciclovir EV + AAS + fotocoagulação com laser de argônio & Série de casos, sem grupo controle \\
\hline Shiraki et al., 1997 & $\begin{array}{l}\text { Aciclovir EV + interferon + VVPP + lensectomia + } \\
\text { fotocoagulação com laser de argônio }\end{array}$ & Relato de caso \\
\hline Amaro, 1993 & $\begin{array}{l}\text { Aciclovir oral + corticosteróides + AAS + fotocoagulação } \\
\text { com laser de argônio }\end{array}$ & Série de casos, sem grupo controle \\
\hline Hirst et al., 1987 & Aciclovir EV + colírio cicloplégico & Série de casos, sem grupo controle \\
\hline Han et al., 1987 & $\begin{array}{l}\text { Fotocoagulação com laser de argônio ou criptônio + } \\
\text { aciclovir + antiagregante plaquetário + corticóide oral }\end{array}$ & Série de casos, sem grupo controle \\
\hline $\begin{array}{l}\text { Immonen, Laatikainen, } \\
\text { Linnanvuori, } 1989\end{array}$ & VVPP + aciclovir EV + corticóide oral & Relato de caso \\
\hline Blumenkranz et al., 1986 & Aciclovir EV + antiagregante plaquetário + corticóide oral & Estudo prospectivo, sem grupo controle \\
\hline Peyman et al., 1984 & VVPP + aciclovir EV + aciclovir intravítreo & Relato de caso \\
\hline
\end{tabular}

\section{DISCUSSÃO}

Não foram encontrados, nesta revisão sistemática, estudos clínicos randomizados que avaliassem o efeito das drogas antivirais preconizadas como eficazes no tratamento da necrose aguda de retina, como aciclovir, ganciclovir, valaciclovir e foscarnet.

A fotocoagulação com laser, como fator de proteção contra a ocorrência de descolamento de retina, foi abordada em 6 estudos, 5 séries de caso e um estudo clínico controlado ${ }^{(16,21-24)}$, sendo que este último foi considerado como apto para preencher os critérios de inclusão, mesmo não sendo randomizado ${ }^{(20)}$.

Quatro estudos consideraram a vitrectomia via pars plana como forma de tratamento para as alterações do vítreo e como prevenção do descolamento de retina, porém nenhum preencheu os critérios para inclusão na revisão, por se tratarem de estudo de casos sem grupo controle c(25-28). $^{\text {. }}$

Dois estudos avaliaram o uso de aciclovir EV no tratamento da necrose aguda de retina, porém não preencheram os critérios para inclusão na revisão ${ }^{(15,29)}$.

$\mathrm{O}$ uso de aciclovir EV atuando como proteção contra a ocorrência de necrose aguda de retina no olho contralateral, foi objeto do estudo de Palay et al. (1991), que apesar de ser um estudo clínico controlado não randomizado, foi considerado como um estudo que preenche os critérios de inclusão nesta revisão ${ }^{(19)}$.

O estudo de Palay et al., 1991, que propõe o tratamento com aciclovir EV como fator de proteção contra o aparecimento da doença no olho contralateral, considerado como um estudo que preenche os critérios de inclusão nesta revisão, é de metodologia fraca, pois é um estudo clínico controlado não randomizado, em que são apresentados pacientes com tempos de acompanhamento diferentes, não se descrevendo as razões pelas quais há essa diversidade de acompanhamento; desfechos, como avaliação da acuidade visual e intensidade da inflamação, não são considerados, como também não foi considerada a evolução da doença no olho afetado; o uso de corticóides não segue um padrão uniforme, nem tampouco são descritas as doses utilizadas em nenhum dos dois grupos estudados.

No entanto o estudo de Palay et al., apesar de sua fragilidade metodológica, mostra que o tratamento proposto exerce um fator de proteção quanto ao acometimento do olho contralateral, conclusão esta baseada na avaliação dos resultados e na análise do gráfico 1 , onde a linha horizontal representa o intervalo de confiança, ou seja, o intervalo no qual a proporção de eventos é observada; neste caso, a ausência de doença no olho contralateral, seria observada em $95 \%$ das vezes, se o estudo fosse repetido 100 vezes e a linha vertical marca o efeito nulo ou risco relativo igual a 1; à esquerda desta linha ficam os resultados que apontam um efeito positivo do tratamento em relação ao grupo controle, e, à sua direita, os resultados que expressam um aumento do risco do tratamento em relação ao controle.

O estudo clínico controlado de Sternberg et al., 1988, que avalia a fotocoagulação com laser de argônio como um fator protetor contra a ocorrência de descolamento de retina na necrose aguda de retina, também apresenta uma metodologia pobre $^{(20)}$. Assim como o estudo de Palay et al., 1991, é um estudo clínico controlado não randomizado. Não esclarece se os pacientes incluídos no trabalho são imunocompetentes ou imunodeprimidos. A intervenção proposta é realizada em momentos diferentes da evolução da doença, o poder e a duração dos pulsos do laser de argônio não foram os mesmos para todos o pacientes do grupo tratado, o grau de acometimento do olho não é o mesmo no momento em que os pacientes 
recebem o tratamento com fotocoagulação com laser de argônio, o tratamento clínico para necrose aguda de retina não é padronizado, as doses de aciclovir EV e de corticóide, quando este é utilizado, não são descritas e os pacientes recebem drogas diferentes por tempo diferenciado, em ambos os grupos. São apresentados pacientes com tempos de acompanhamento diferentes, não se descrevendo as razões pelas quais isto acontece.

Fazendo a mesma análise em relação ao gráfico 2, verificamos que o resultado está posicionado à esquerda da linha vertical (risco relativo igual a 1), concluindo-se assim que, apesar de apresentar também metodologia deficiente, o estudo mostra que o tratamento exerce um fator de proteção quanto ao desenvolvimento de descolamento de retina.

Comparando os gráficos 1 e 2, observamos que o resultado do primeiro estudo se posiciona mais à esquerda em relação à linha vertical que o resultado do segundo estudo, demonstrando assim maior representatividade estatística do primeiro estudo $^{(19)}$, em relação ao segundo ${ }^{(20)}$.

\section{CONCLUSÃo}

Apesar de ambos os estudos incluídos na revisão sistemática serem de metodologia muito fraca, decidimos mantê-los pois se constatou uma carência quase absoluta de trabalhos com grau de evidência suficiente para se estabelecer a conduta no tratamento desta patologia, que apresenta morbidade bastante elevada.

$\mathrm{O}$ autor constata que há falta de evidências que suporte o tratamento para a Necrose Aguda de Retina.

Evidências fracas mostram que o uso de aciclovir pode exercer um papel de proteção contra a ocorrência de doença bilateral, assim como evidências fracas demonstram a possibilidade da fotocoagulação com laser de argônio prevenir o descolamento de retina nos olhos acometidos pela doença.

Estudos clínicos randomizados são necessários para se estabelecer uma conduta no tratamento da necrose aguda de retina.

\section{ABSTRACT}

Purpose: The purpose of this study was to identify, according to an sistematic review, the best treatment for acute retinal necrosis. Methods: Following the methodologic guidance of the Cochrane Collaboration and its editorial subgroup "Eye and Vision Group", using search strategy for study identification, articles about the treatment of acute retinal necrosis were selected. Results: 146 bibliographic references were selected, 13 studies were considered relevant. Among them 2 were considered to follow the inclusion criteria. The first study tested the possibility of using intravenous acyclovir associated with corticosteroids, protecting contralateral eye from being affected. Fifty-four patients who reported unilateral acute retinal necrosis have been studied, 31 treated and 23 not treated, the disease occurring in the contralateral eye in $12.9 \%$ of the patients of the treated group and in $69.5 \%$ of the patients in the non-treated group. The second study considered treatment with photocoagulation with argon laser to prevent retinal detachment, that was observed in $57.1 \%$ of the eyes. The second included study disclosed 19 eyes affected by acute retinal necrosis, considering that 12 of these eyes were treated with photocoagulation with argon laser to prevent retinal detachment. Among the 12 eyes, 2 developed retinal detachment (16.6\%) while, in the non-treated group, 7 eyes, 4 developed retinal detachment $(57.1 \%)$. Conclusion: The author concludes that both types of proposed interventions have proven to be effective. However, once the mentioned studies are methodologically considered weak, it is necessary to perform randomized clinical trials with the purpose of establishing the most suitable treatment for acute retinal necrosis.

Keywords: Retinal necrosis syndrome acute/drug therapy; Retina/pathology; Acyclovir/therapeutic use; Evidence Based Medicine; Treatment outcome; Meta-analysis

\section{REFERÊNCIAS}

1. Culbertson WW, Clarkson JG, Blumenkranz M, Lewis ML. Acute retinal necrosis. Am J Ophthalmol. 1983;96(5):683-5.

2. Urayama A, Yamada N, Sasaki T. Unilateral acute uveitis with periarteritis and detachment. Jpn J Clin Ophthalmol. 1971;25:607-19.

3. Willerson D Jr, Aaberg TM, Reeser FH. Necrotizing vaso-occlusive retinitis Am J Ophthalmol. 1977;84(2):209-19.

4. Young NJ, Bird AC. Bilateral acute retinal necrosis: a case report. Br J Ophthalmol. 1978;62(9):581-90.

5. Hayreh MMS, Kreiger AE, Straatsma BR. Acute retinal necrosis. In: Annual Meeting of the Association for Research and Vision in Ophthalmology; 1980; Fort Lauderdale. Proceedings. Rockville: ARVO; 1980.

6. Sato EH, Machado VHT, Farah Neto ME, Abreu MT, Belfort Júnior R. Necrose aguda de retina. Rev Bras Oftalmol. 1985;4(1):28-31.

7. Amaro MH, Amaro JH. Necrose retiniana aguda manuseio de um caso. Rev Bras Oftalmol. 1987;46(1):30-2.

8. Medeiros HAG, Medeiros JEG. Considerações sobre retinite a citomegalovírus e síndrome de necrose retiniana aguda. Rev Bras Oftalmol. 1991;50(1):33-8.

9. Lacerda RR. Necrose retiniana aguda estudo clínico de doze casos. Rev Bras Oftalmol. 1993;52(3):23-33.

10. Nussenblatt RB, Whitcup SM, Palestine AG. Acute retinal necrosis and progressive outer retinal necrosis. In: Nussenblatt RB, Palestine AG, Whitecup SM, editors. Uveitis: fundamentals and clinical practice. Saint Louis: MosbyYear Book; 1996. p.198-206.

11. Holland GN. Standard diagnostic criteria for the acute retinal necrosis syndrome. Executive Committee of the American Uveitis Society. Am J Ophthalmol. 1994;117(5):663-7.

12. Forster DJ, Dugel PU, Frangieh GT, Liggett PE, Rao NA. Rapidly progressive outer retinal necrosis in the acquired immunodeficiency syndrome. Am J Ophthalmol. 1990;110(4):341-8.

13. Fisher JP, Lewis ML, Blumenkranz M, Culbertson WW, Flynn HW Jr, Clarkson JG, et al. The acute retinal necrosis syndrome. Part 1: Clinical manifestations. Ophthalmology. 1982;89(12):1309-16.

14. Freitas NA, Oréfice F. Uveítes virais. In: Oréfice F. Uveíte clínica e cirúrgica Rio de Janeiro: Cultura Médica; 2000. p.461-95.

15. Blumenkranz MS, Culbertson WW, Clarkson JG, Dix R. Treatment of the acute retinal necrosis syndrome with intravenous acyclovir. Ophthalmology. 1986;93(3):296-300.

16. Luu KK, Scott IU, Chaudhry NA, Verm A, Davis JL. Intravitreal antivira injections as adjunctive therapy in the management of immunocompetent patients with necrotizing herpetic retinopathy. Am J Ophthalmol. 2000;129(6): 811-3.

17. Belfort Júnior R, Muccioli C. Retinal detachment secondary to herpes retinitis. 
In: Boyd BF, Boyd S, editors. Retinal and vitreoretinal surgery: mastering the latest techniques. Panamá: Highlights of Ophthalmology International; 2002. p.485-96.

18. Clarke M, Oxman $\mathrm{AD}$, editors. Cochrane reviewers' handbook 4.1.6 [text on the Internet]. [cited 2003 Jan 31]. Available from: http://www.cochrane.dk/ cochrane/handbook/handbook.htm. Updated Jan 2003.

19. Palay DA, Sternberg P, Davis J, Lewis H, Holland GN, Mieler WF, et al Decrease in the risk of bilateral acute retinal necrosis by acyclovir therapy. Am J Ophthalmol. 1991;112(3):250-5.

20. Sternberg P Jr, Han DP, Yeo JH, Barr CC, Lewis H, Williams GA, et al. Photocoagulation to prevent retinal detachment in acute retinal necrosis. Ophthalmology. 1988;95(10):1389-93.

21. Aslanides IM, De Souza S, Wong DT, Giavedoni LR, Altomare F, Detorakis ET, et al. Oral valacyclovir in the treatment of acute retinal necrosis syndrome. Retina. 2002;22(3):352-4.

22. Hudde T, Althaus C, Sundmacher R. [Acute retinal necrosis syndrome. Argon laser coagulation for prevention of rhegmatogenic retinal detachment]. Ophthalmologe. 1998;95(7):473-7. Germany.
23. Amaro MH. Fotocoagulação profilática na necrose retiniana aguda. Rev Bras Oftalmol. 1993;52(1):53-6.

24. Han DP, Lewis H, Williams GA, Mieler WF, Abrams GW, Aaberg TM. Laser photocoagulation in the acute retinal necrosis syndrome. Arch Ophthalmol. 1987;105(8):1051-4.

25. Muller B, Velhagen KH, Pleyer U. [Acute retinal necrosis syndrome: analysis, therapy and long-term follow up of 14 eyes]. Klin Monatsbl Augenheilkd. 2000;217(6):345-50. Germany.

26. Shiraki K, Moriwaki M, Ataka S, Henmi K, Miki T, Kanaoka Y. Acute retinal necrosis late in the second trimester. Am J Ophthalmol. 1998;125(1):103-4.

27. Immonen I, Laatikainen L, Linnanvuori K. Acute retinal necrosis syndrome treated with vitrectomy and intravenous acyclovir. Acta Ophthalmol (Copenh). 1989;67(1):106-8.

28. Peyman GA, Goldberg MF, Uninsky E, Tessler H, Pulido J, Hendricks R. Vitrectomy and intravitreal antiviral drug therapy in acute retinal necrosis syndrome: report of two cases. Arch Ophthalmol. 1984;102(11):1618-21.

29. Hirst LW, Beyer TL, Waters D, Fleischman J. Successful management of acute retinal necrosis with intravenous acyclovir. Ann Ophthalmol. 1987;19(12):445-8.

\section{1 a REUNIÃO DO CENTRO DE ESTUDOS “CYRO DE REZENDE"}

\section{3 e 04 de Dezembro}

Centro de Convenções de Ribeirão Preto

\section{Ribeirão Preto - SP}

IN FO RMAÇÕES: Tel.: (11) 3361-3056

Fax: (11) 3361-3089

E-mail: oftalmo@eventus.com.br

Home-page: www.eventus.com.br/ oftalmo 